\title{
Selective attention effects on retinal and forebrain responses in humans: A replication and extension
}

\author{
ROBERT G. EASON \\ University of North Carolina, Greensboro, North Carolina
}

\begin{abstract}
Eason, Oakley, and Flowers (1983) reported that the amplitude of the b-wave and afterpotential of the electroretinogram is affected by the attentional state of the subject. Two follow-up studies were conducted primarily to replicate their findings. The composite results confirmed those previously reported, providing further evidence for early sensory filtering of irrelevant, unattended information. The results also provide additional evidence for the existence of retinopetal fibers in humans.
\end{abstract}

A question of continuing interest to behavioral neuroscientists is whether changes in the attentional state of an organism differentially affect sensory transmission precortically (Hillyard \& Picton, 1979; Lindsley, 1959; Livingston, 1978; Näätänen, 1982). Although many animal studies have provided evidence for response attenuation or enhancement in sensory pathways during selective attention (Goldberg \& Wurtz, 1972; Livingston, 1978; Oatman \& Anderson, 1980), very few studies have provided evidence for such effects in humans (Eason, Oakley, \& Flowers, 1983; Lukas, 1981).

Hillyard and associates (Hillyard \& Picton, 1979) detected no differences in either the amplitude or latency of cochlear nerve and brain-stem auditory evoked potentials (AEPs) during selective attention. Lukas (1980, 1981), in later AEP experiments utilizing more effective selective attention manipulations, more subjects, and tone stimuli rather than clicks, observed both amplitude and latency differences in the cochlear nerve response as well as in the brain stem. Eason et al. (1983), working with the visual modality, demonstrated that the amplitudes of the b-wave and afterpotential of the electroretinogram (ERG) also are affected by one's attentional state. They interpreted their results as providing support for the hypothesis that sustained voluntary attention to locations in space is mediated partly by centrally controlled centrifugal pathways that differentially modify synaptic transmission at a precortical level.

The latter study has received mixed reactions, partly because many cognitive neuroscientists conceptualize attentional processes within the context of informationprocessing models that assume that selective filtering of

This research was supported in part by UNC-G RC Grant 7612 and was presented at the EPA meetings in Baltimore, April 1984. The author expresses his gratitude to the students who served as subjects. The author's mailing address is: Department of Psychology, University of North Carolina, Greensboro, NC 27412. irrelevant events requires stimulus-related decisions involving the cerebral cortex (Näätänen, 1982), and partly because some neuroscientists consider it unlikely that the human optic nerve contains retinopetal fibers (e.g., Rodieck, 1973). In view of the existing skepticism, two follow-up experiments have been conducted in an effort to replicate and extend the findings of the Eason et al. (1983) study.

Each follow-up experiment reported here utilized one of the two attention manipulations employed in the 1983 study. In Experiment 1, subjects were instructed to attend and respond to stimuli appearing peripherally in a given (relevant) visual field (e.g., right) while attempting to ignore those appearing concomitantly at a homologous location in the opposite (irrelevant) field, and vice versa. In Experiment 2, subjects were instructed to attend and respond to stimuli appearing peripherally in the left (relevant) visual field while attempting to ignore foveally presented (irrelevant) stimuli, and vice versa. In the 1983 study, the foveal-peripheral manipulation produced a more pronounced differential response at the level of the retina than did the peripheral-peripheral manipulation. A similar effect was observed in occipitally recorded visual evoked responses in an earlier study (Eason, 1981). Thus, Experiment 1 constituted a relatively rigorous test of the robustness of the retinal attention effect, whereas Experiment 2 constituted a more sensitive test of the reliability of the effect.

To gain further insight into the locus of early filtering in the visual system during selective attention, recordings were obtained from new sites as well as from those used in the 1983 study.

\section{EXPERIMENT 1}

\section{Method}

Sixteen advanced undergraduate students, four men and eight women, served as subjects. Those who usually wore prescription lenses did so during data collection.

Each subject participated in four trials in a $2-\mathrm{h}$ session. 
Attention was directed toward the left-visual-field (LVF) location on two trials and toward the right (RVF) location on the other two. A 10-min break occurred midway through the session.

The stimuli consisted of 6.5-deg circular patches of flashed blue light of 10-microsec duration. The flashes occurred concomitantly, but never simultaneously, 30 deg peripherally in each field on a red tangent screen (relative to the horizontal axis) located $40 \mathrm{~cm}$ from the stimulated eye. The blue stimuli were imposed on a red background to maximize the rod response while minimizing that of the cones. Flash intensity was about $3.5 \mathrm{log}$ units above a background level of approximately $2 \mathrm{~mL}$.

One hundred single flashes were presented from within each visual field on each trial. Randomly interspersed among the single flashes were doublets (two flashes occurring $200 \mathrm{msec}$ apart) that occurred, on the average, $25 \%$ of the time. The interstimulus interval varied from a minimum of $800 \mathrm{msec}$ up to about $2 \mathrm{sec}$ in a manner approximating a j-shaped distribution. The visual field within which each successive flash occurred was randomized.

For the relevant field, the subject responded to each doublet by lifting his or her finger from a key. If the time to respond exceeded $250 \mathrm{msec}$, a beeper signaled failure to meet criterion.

The key used for responding to doublets also served as the master switch for activating a programmer that controlled stimulus delivery. Thus, the subject could start or stop the stimulus delivery system by depressing or releasing the key. Prior to data collection, the subject practiced discriminating between single and double flashes and responding to the latter. Practice continued until he or she could perform the task for periods of 8-10 sec without blinking or moving the eyes.

The head was positioned with a chinrest. To reduce forehead muscle potential artifacts, the fixation point was positioned $10 \mathrm{deg}$ below eye level. Eye blinks and movements were monitored with a polygraph. To minimize such artifacts, the subject was encouraged to take frequent breaks and to engage in such activity only during those breaks.

Two sets of retinal recordings were obtained with electrodes placed (1) at the internal canthus of the right eye relative to the right earlobe $\left(\mathrm{IC} / \mathrm{A}_{2}\right)$, as in the 1983 study, and (2) at the internal canthus of the same eye relative to the external canthus (IC/EC). An electrode on the left earlobe served as ground. Only the right eye was stimulated.

Following amplification with band-pass filters set at 1 and $35 \mathrm{~Hz}$, retinal responses were averaged on line and recorded with an $\mathrm{x}-\mathrm{y}$ plotter. Each average was based on 100 single-flash presentations. Responses to doublets were not included. While recording, the subject sat in an electrically shielded room. White noise masked extraneous sounds.

\section{Results}

Group analog averages of the responses to relevant (solid lines) and irrelevant (dotted lines) stimuli are shown in Figure 1. Each tracing is a grand average of 32 individual averaged responses obtained from 16 subjects ( 2 replications/condition/subject) and is based on a total of 3,200 stimulus presentations (100/individual average). The a-wave, $b$-wave, and afterpotential are identified in the top row of tracings.

To determine whether the apparent attention effects manifested in the tracings were statistically significant, correlated $t$ tests were performed on measures obtained from individual recordings (collapsed across replications) of the 16 subjects for each experimental condition. The amplitude of the b-wave was quantified by measuring the vertical distance between the peak falling within a 20-30-msec window of each individual average (b-wave

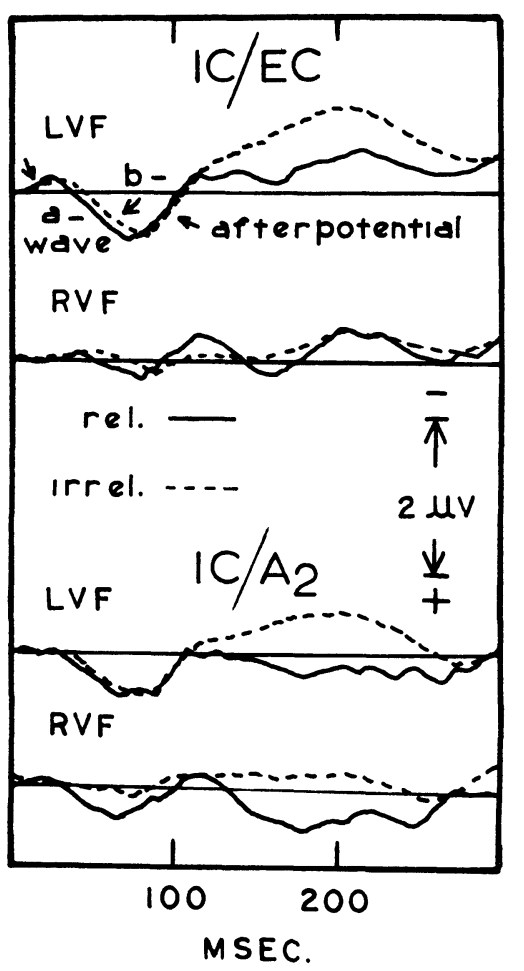

Figure 1. Grand averages of retinal responses obtained from right and left visual fields with two electrode montages (IC/EC and IC/ $\AA_{2}$ ) under relevant and irrelevant conditions.

onset) and the lowest point falling within a $60-80-\mathrm{msec}$ window (b-wave offset). The amplitude of the afterpotential was quantified by measuring the vertical distance between the offset of the b-wave (afterpotential onset) and the highest negative peak falling within a 100-130msec window (offset). The peak latency of the b-wave also was measured for those subjects in whom it could be discerned.

Visual field relevancy influenced some, but not all, of the measures. Neither the amplitude of the b-wave nor afterpotential of responses evoked by LVF stimuli, whether recorded at IC/EC or IC/ $\mathbf{A}_{2}$, was significantly affected by the attention manipulation. In marked contrast, the IC/ $\mathrm{A}_{2}$ responses evoked by RVF stimuli were significantly larger when that field was relevant [for the $b$-wave, $t(1,15)=2.20, p<.05$; for the afterpotential, $\mathrm{t}(1,15)=3.58, \mathrm{p}<.01]$. Also, the amplitude of the afterpotential component of the IC/EC responses to RVF stimuli was significantly greater when that field was relevant $[t(1,15)=3.20, p<.05]$, but the amplitude of the $b$-wave component was not significantly affected. An analysis performed on the combined b-wave and afterpotential amplitude measures of IC/EC responses to RVF stimuli with respect to relevancy barely missed significance at the .05 level $[t(1,15)=$ $2.03, \mathrm{p}<.06]$.

An amplitude analysis performed on composite measures averaged across the $b$-wave and afterpotential, the two visual fields, and the two recording sites indi- 
cated that the composite response was greater under the task-relevant condition $[\mathrm{t}(1,15)=2.45, \mathrm{p}<.05]$. A similar analysis performed on the b-wave data only, after averaging across the two sets of recordings and visual fields, revealed a significantly greater amplitude for the task-relevant condition $[\mathrm{t}(1,15)=2.13, \mathrm{p}<.05]$.

An analysis of b-wave peak latency data (collapsed across visual fields) for eight subjects from whom measurements were obtainable resulted in a significantly shorter latency under the task-relevant condition for the $\mathrm{IC} / \mathrm{EC}$ recordings $[\mathrm{t}(1,7)=6.57, \mathrm{p}<.01]$ as well as for the $\mathrm{IC} / \mathrm{A}_{2}$ recordings $[\mathrm{t}(1,7)=7.05, \mathrm{p}<.01]$. Peak latencies of the afterpotential could not be measured with confidence for enough subjects to permit analysis.

\section{EXPERIMENT 2}

\section{Method}

Thirty-two adults, 12 men and 18 women, participated. Twenty-eight were advanced undergraduate students, 3 were graduate students, and 1 was a faculty member. Those who usually wore lenses did so during data collection.

The design was identical to that of Experiment 1, except that attention was directed toward LVF stimuli on two trials and toward foveal stimuli on the other two. Recordings always were obtained to the LVF stimuli regardless of which location (LVF or fovea) was relevant.

With the exceptions noted here, the equipment and procedures were identical to those of Experiment 1. Randomly interspersed doublets occurred $12.5 \%$ of the time among singleflash stimuli appearing in the LVF. In addition to responding to the doublets, the subjects counted the number of flashes presented during a trial, including doublets.

The foveal stimuli used here were described in detail in the 1983 study. They consisted of rectangular displays of 12 typewritten numerals arranged in four columns and three rows. The displays were presented at a rate of $1 / \mathrm{sec}$ when the subject depressed the master key. When the foveal task was relevant, the subjects counted the number of times a particular numeral (e.g., 3) appeared in a sequence of displays.

To minimize head movements, the subjects placed their teeth lightly against a biteboard. To minimize eye blinks and movements, the experimenter maintained control over the workbreak cycle rather than leaving it up to the subjects, as in Experiment 1 . On those trials in which the LVF location was relevant, the subjects, either on cue from the experimenter or after every doublet, took a break about every 6-8 sec. Each break lasted 3-5 sec, during which time the subjects blinked and moved their eyes. The task was resumed on cue. About 15-20 breaks were taken per trial. A similar work-break cycle was utilized during the foveal task. These frequent work-break cycles significantly reduced the incidence of blinks and movements compared with that of Experiment 1.

Retinal recordings were obtained with an electrode placed at the internal canthus of the right eye relative to the right earlobe. Frontal scalp recordings were obtained with electrodes placed at $\mathrm{Fp}_{2}$ and $\mathrm{F}_{4}$. Both eyes were open during data collection.

\section{Results}

Group analog averages of retinal (IC/ $\left.\mathrm{A}_{2}\right)$ and frontal scalp recordings $\left(\mathrm{Fp}_{2} / \mathrm{F}_{4}\right)$ to the LVF stimuli when relevant and when irrelevant are shown in Figure 2. Each tracing is a grand average of 64 individual averaged

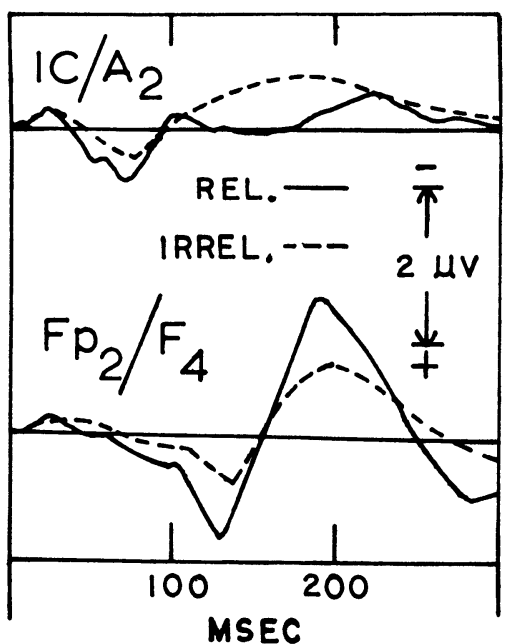

Figure 2. Grand averages of retinal responses (IC/ $\left.\mathrm{A}_{2}\right)$ and frontal scalp recordings $\left(\mathrm{Fp}_{2} / \mathbf{F}_{4}\right)$ obtained to left visual field stimulation under relevant and irrelevant conditions.

responses obtained from 32 subjects ( 2 replications/ condition/subject) and is based on a total of 6,400 stimulus presentations (100/individual average).

ERG. The a-wave, b-wave, and afterpotential are readily apparent in the top row of tracings. It is also apparent that the amplitudes of both the $b$-wave and the afterpotential were larger when the LVF was relevant. Closer inspection reveals that the peak latency of both of these components was shorter by about $5 \mathrm{msec}$ for the task-relevant condition. Further inspection reveals that late deflection beginning at the cessation of the afterpotential (at about $110 \mathrm{msec}$ ) and terminating at about $220 \mathrm{msec}$ was more positive under the task-relevant condition. Maximum separation occurred at about $160 \mathrm{msec}$.

To test for statistical significance, measures of amplitude and latency of the b-wave and the afterpotential, along with amplitude measures of the late deflection, were obtained for each condition (collapsed across replications) of the 32 subjects. A correlated t test revealed that the b-wave was significantly larger for the taskrelevant condition $[t(1,31)=2.83, p<.01]$. A comparable test performed on $b$-wave latency of 24 subjects for whom it was measurable was significantly shorter under the task-relevant condition $[\mathrm{t}(1,23)=3.81$, $\mathrm{p}<.001]$. An analysis of the afterpotential amplitude measures revealed a significantly greater amplitude when the LVF was relevant than when it was irrelevant $[t(1,31)=3.36, p<.01]$. Likewise, the afterpotential peak latency of those subjects in whom it was discernible was shorter when the LVF was task relevant $[t(1,22)$ $3.41, \mathrm{p}<.01]$. A t test performed on the late potential measures at $160 \mathrm{msec}$ revealed that the polarity difference between the relevant and irrelevant conditions was significant $[t(1,31)=4.05, \mathrm{p}<.001]$.

Frontal scalp recordings. The grand averages of the frontal scalp recordings (lower tracings of Figure 2) 
reflect an initial negativity peaking at $30 \mathrm{msec}$ for the relevant and at $40 \mathrm{msec}$ for the irrelevant conditions $\left(\mathrm{N}_{30 / 40}\right)$. This negativity is followed by a low-amplitude positive deflection that levels off or slightly reverse direction at $100 \mathrm{msec}$ for the relevant and at $105 \mathrm{msec}$ for the irrelevant LVF conditions $\left(\mathrm{P}_{100 / 105}\right)$. The amplitude of this positive component was significantly greater under the relevant condition $[\mathrm{t}(1,31)=2.50$, $\mathrm{p}<.02$ ], and its offset latency, based on measurements of 30 subjects, was shorter $[\mathrm{t}(1,29)=3.34, \mathrm{p}<.001]$. $\mathrm{P}_{100 / 105}$ is followed by a sharp positivity that peaks at $130 \mathrm{msec}$ for the relevant and at $135 \mathrm{msec}$ for the irrelevant conditions $\left(\mathrm{P}_{130 / 135}\right)$. Its amplitude was significantly larger for the relevant condition $[\mathrm{t}(1,31)=$ $5.50, \mathrm{p}<.001]$, and its peak latency, based on measures of 24 subjects, was shorter $[\mathrm{t}(1,23)=4.12, \mathrm{p}<.001]$.

The amplitude of the large negative wave $\left(\mathrm{N}_{190}\right)$ that follows $\mathrm{P}_{100 / 105}$ was larger for the relevant condition $[t(1,31)=5.68, p<.001]$, but its latency was not significantly affected.

\section{DISCUSSION}

In general, the effect of stimulus location relevancy on ERG responses obtained in these follow-up experiments indicates that the findings reported in the 1983 study can be replicated. Yet it is clear that the effect is a delicate one. In Experiment 1, demonstration of the effect varied with visual field and recording site, being most evident for the RVF and the EC/ $\mathrm{A}_{2}$ recordings. In contrast to Experiment 1 , in which an insignificant effect was obtained for the LVF, Experiment 2 revealed that the amplitudes of both the b-wave and the afterpotential were greater and that their peak latencies were shorter when the LVF was relevant than when it was irrelevant. The amplitude effects are consistent with those observed for the LVF in the 1983 study. Effects on latency were not reported in the 1983 study, but a subsequent analysis of the b-wave peak latency measures of 30 subjects (the latencies of 2 subjects were immeasurable) revealed significantly shorter latencies when a given field was relevant than when it was irrelevant $[\mathrm{F}(2,28)=11.50, \mathrm{p}<.01]$.

The clearer demonstration of the relevancy effect in Experiment 2 than in Experiment 1 may be attributed (1) to a more effective means of varying selective attention (foveal-peripheral rather than peripheral-peripheral shifts), (2) to utilizing twice as many subjects, and (3) to maintaining tighter control over the work-break cycles during each trial. Keeping the work-break cycles short and consistent within and across subjects may have sharpened the subjects' capacity to selectively attend during each work interval. Also, tight control over these cycles lessened the degree to which eye blinks, movements, and muscle potential artifacts generated error variance.

The effects of location relevancy on the IC/EC recordings in Experiment 1 were less clear than those on the IC/ $\mathrm{A}_{2}$ recordings. It was thought that the IC/EC recordings may have reflected the ERG in "purer" form than did the IC/A $\mathrm{A}_{2}$ recordings, since the $\mathrm{A}_{2}$ electrode was farther from the eye. However, examination of individual averaged responses, as well as the group averages of Figure 1, revealed the presence of late oscillations believed to be of cortical origin that were more pronounced in the IC/EC than in the $I C / A_{2}$ recordings. This suggests that the earlobe was a less active site than the external canthus for recording the ERGs and that the IC/ $\mathrm{A}_{2}$ montage gave a more accurate reflection of the attention location effect.

The source of origin of the neural generators responsible for voltages recorded at $\mathrm{Fp}_{2} / \mathrm{F}_{4}$ cannot be established with confidence without further study. The deflections occurring over the first $60 \mathrm{msec}$ have about the same latencies and are of the same polarity as the ERG, although much reduced in amplitude. The
$P_{130 / 135}$ deflection has an onset latency comparable to that recorded from single neurons in the frontal eye field of monkeys (Goldberg \& Bushnell, 1981) and may reflect neural activity in that area.

In conclusion, the results of these follow-up experiments, in addition to replicating the 1983 study, provided further evidence for early sensory filtering of irrelevant, unattended information. As Lukas $(1980,1981)$ observed for the auditory system, the detection of such filtering through the use of averaged evoked potential procedures is at best problematic. To demonstrate the effect, one must utilize procedures that optimize the selective attention process and that maximize differential transmission of impulses through those pathways activated by relevant and irrelevant stimuli. We have obtained clearest results for the visual system when we have used relatively large numbers of highly motivated subjects performing demanding discrimination tasks combined with a memory requirement, and when we have maintained tight control over work-break cycles during each run.

The results reported here also provide further evidence for the existence of retinopetal fibers in humans. ${ }^{1}$

\section{REFERENCES}

EAson, R. G. (1981). Visual evoked potential correlates of early neural filtering during selective attention. Bulletin of the Psychonomic Society, 18, 203-206.

Eason, R. G., OAkLey, M., \& Flowers, L. (1983). Central neural influences on the human retina during selective attention. Physiological Psychology, 11, 19-28.

Goldberg, M. E., \& Bushnell, M. C. (1981). Behavioral enhancement of visual responses in monkey cerebral cortex. II. Modulation in frontal eye fields specifically related to saccades. Journal of Neurophysiology, 46, 773-787.

GoldberG, M. E., \& Wurtz, R. H. (1972). Activity of superior colliculus in behaving monkey. II. Effects of attention on neuronal responses. Journal of Neurophysiology, 35, 560-574.

Hilly ard, S. A., \& Picton, T. W. (1979). Event-related potentials and selective information processing in man. In J. E. Desmedt (Ed.), Cognitive components in cerebral event-related potentials and selective attention: Progress in clinical neurophysiology (Vol. 6). Basel, Switzerland: Karger.

ITAYA, S. K. (1980). Retinal efferents from the pretectal area in the rat. Brain Research, 201, 436-441.

LindsLeY, D. B. (1959). Attention, consciousness, sleep and wakefulness. In J. Field (Ed.), Handbook of physiology: Vol. 1. Neurophysiology. Washington, DC: American Physiological Society.

Livingston, R. B. (1978). Sensory processing, perception and behavior. New York: Raven Press.

LUKAs, J. H. (1980). Human auditory attention: The olivocochlear bundle may function as a peripheral filter. Psychophysiology, 17, 444-452.

LuKAs, J. H. (1981). The role of efferent inhibition in human auditory attention: An examination of the auditory brainstem potentials. International Journal of Neuroscience, 12, 137-145.

NÄÄtÄnen, R. (1982). Processing negativity: An evoked-potential reflection of selective attention. Psychological Bulletin, 92 , 605-640.

Oatman, L. C., \& Anderson, B. W. (1980). Suppression of the auditory frequency following response during visual attention. Electroencephalography and Clinical Neurophysiology, 49, 314-322.

RoDieck, R. W. (1973). The vertebrate retina. San Francisco: Freeman.

\section{NOTE}

1. See Eason et al. (1983) and Livingston (1978) for recent reviews. An HRP rat study by Itaya (1980), not reported in earlier reviews, provides additional anatomical evidence for the existence of retinopetal fibers in mammals.

(Manuscript received for publication March 14, 1984.) 